\title{
PRIMER ESTUdiO GENÉTICO DE LA NUTRIA MARINA (LONTRA FELINA) EN LA COSTA PERUANA
}

\section{FIRST GENETIC STUDY OF THE MARINE OTTER (LONTRA FELINA) AT THE PERUVIAN COAST}

\author{
JuAn VAlQui \\ Centro de Ornitología y Biodiversidad (CORBIDi), Calle Santa Rita io5, Of. 202, Lima 33, Perú \\ Centro Peruano de Estudios Cetológicos (CEPEC), Calle Jorge Chávez 302, Pucusana, Perú
}

\begin{abstract}
RESUMEN
A partir de muestras de heces colectadas en agosto y setiembre del 2008 en el rango de distribución peruano de la nutria marina (Lontra felina), se realizó un análisis del ADN mitocondrial de 24 individuos, que mostraron una variabilidad genética relativamente alta (11 haplotipos, $\mathrm{h}=0.86$ y $\pi=0.0117$ ). El estudio representa el primer registro de secuencias genéticas de la especie. La adaptación de esta especie amenazada a un hábitat naturalmente fragmentado podría explicar por qué, a pesar del decrecimiento de la población a causa de los efectos negativos de las actividades humanas en la costa peruana en las últimas décadas, la especie aún mantiene una variabilidad relativamente alta. Sin embargo, de acuerdo a las predicciones de la continua reducción de la población de nutria marina y debido al constante incremento de las amenazas de su población por actividades humanas, se recomienda realizar estudios que profundicen los conocimientos sobre la estructura genética de la especie y se sugiere el establecimiento de por lo menos un área natural protegida en el sur del país, donde se han registrado regiones con elevado número de individuos.
\end{abstract}

Descriptores: nutria marina, genética no invasiva, especie amenazada

\begin{abstract}
Scats from marine otter (Lontra felina) were collected in August and September 2008 along the species 'distribution at the Peruvian coast. Mitochondrial DNA analysis from 24 individuals yielded the first registered sequences of this species, showing relatively high variability (11 haplotypes, $h=0.86$ and $\pi=0.0117)$. Adaptation of this species to a naturally fragmented habitat could explain why, despite the decreasing population number due to negative effects of human activity at the Peruvian coast in the last decades, the species remains with a relatively high variability. According to predictions of continuous decrease in population numbers and constantly increasing threats due to human activity, further studies deepening the knowledge about genetic structuring of the species' population and the establishment of at least one protected area at the south of Peru, where regions with high number of individuals have been registered, are recommended.
\end{abstract}

Keywords: marine otter, non-invasive genetics, endangered species 


\section{INTRODUCCIÓN}

La nutria marina (Lontra felina) (fig. 1), el mamífero marino más pequeño del mundo, habita el litoral rocoso a lo largo de la costa del Pacífico sudamericano, desde la localidad de Chimbote $\left(9^{\circ} \mathrm{S}\right)$ en Perú, hasta la Tierra del Fuego (56 $6^{\circ}$ ) en Chile y Argentina (fig. 2). Su distribución es fragmentada $[1,2]$, debido a que en el litoral se alternan regiones de playas arenosas con zonas rocosas. En el Perú, de los $1300 \mathrm{~km}$ que se encuentran dentro de su rango de distribución, aproximadamente $300 \mathrm{~km}$ representan un hábitat potencial para la especie y las densidades pueden variar entre 0,5 y 10 individuos por kilómetro de litoral rocoso [3, 4]. El rango de distribución individual es menor a cinco kilómetros [5].

La distribución histórica de la nutria marina se ha reducido en las últimas décadas debido a los efectos de la actividad humana, tal como la caza dirigida, la captura incidental y la contaminación, así como por la presencia de perros, gatos y ratas que acompañan a las poblaciones humanas, desplazando a las nutrias de su hábitat $[3,6]$. En el extremo sur de su distribución (Tierra del Fuego), las nutrias casi han desaparecido [2, 3, 7]. Por ello la especie es considerada en peligro de extinción por la Lista Roja de la Unión Internacional para la Conservación de la Naturaleza (International Union for Conservation of Nature - IUCN). Asimismo, figura en los Apéndices I (especies con la mayor amenaza) de la Convención sobre el Comercio Internacional de Especies Amenazadas de Fauna y Flora Silvestres (Convention on International Trade in Endangered Species of Wild Fauna and Flora - CITES) y la Convención sobre las Especies Migratorias (Convention on Migratory Species - CMS). La especie también es protegida por las legislaciones peruana, chilena y argentina por ser considerada una especie amenazada por las respectivas autoridades.

En el Perú, los pocos estudios sobre la especie se han concentrado en censar y evaluar las poblaciones, obteniendo resultados muy divergentes, pero consistentes en cuanto al bajo número de individuos. El estudio de Castilla y Bahamondes [8] (en 1978) revela números poblacionales de 200-300 individuos, mientras Apaza et al. [3] (en el 2003) estimaron la población en unos

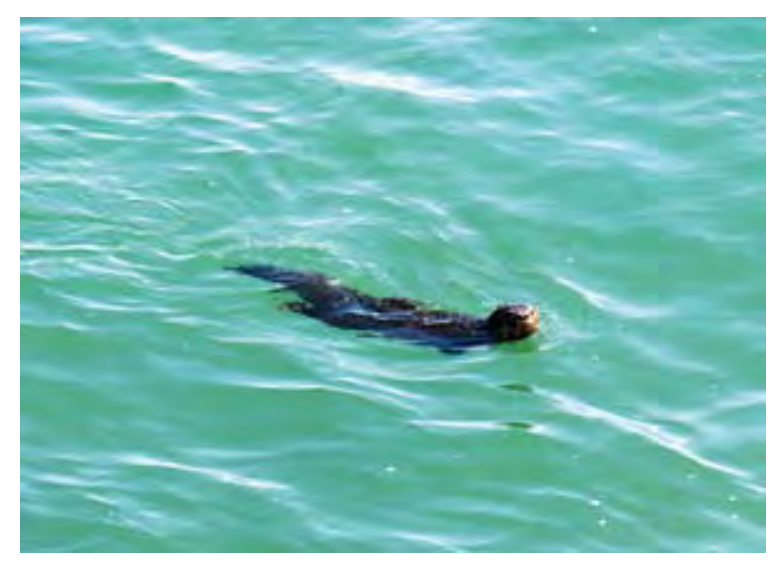

Figura 1: La nutria marina (Lontra felina).

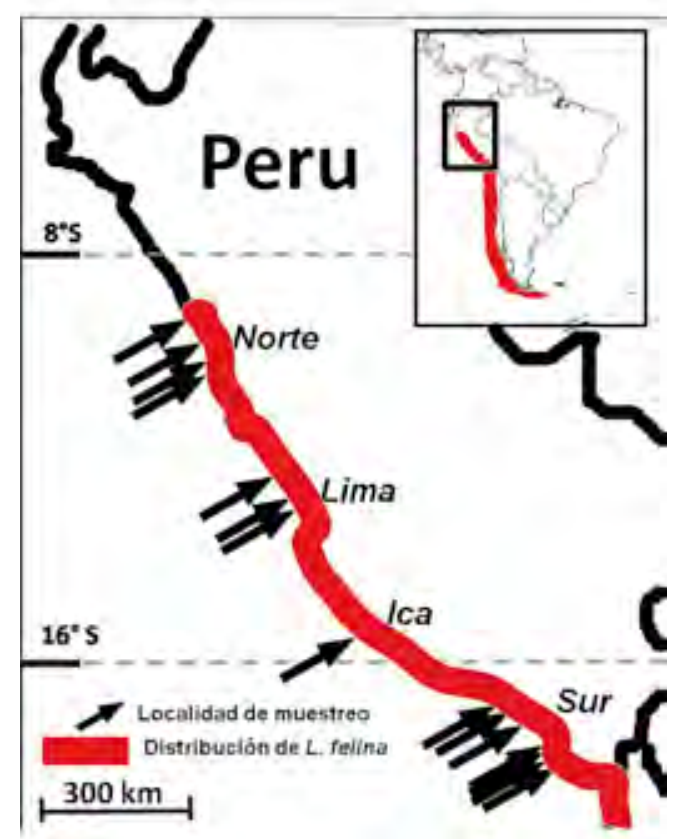

Figura 2: Distribución de L. felina y localidades de muestreo.

700 individuos. Un estudio estimó la población total (para Perú, Chile y Argentina) en 1000 individuos, aunque los investigadores advierten que el valor es probablemente una subestimación [9]. Todos los estudios en Perú, Chile y Argentina sugieren que las amenazas se están incrementando constantemente y presentarán un peligro para la supervivencia de la especie en el futuro [2]. Es por ello que urgen medidas de conservación a lo largo de toda su distribución, para lo cual es relevante basarse en estudios genéticos que determinen el nivel de la variabilidad genética y las características de la estructura poblacional.

El presente estudio, que cubre la distribución peruana de la especie (fig. 2), pretende cerrar esta 
brecha brindando los primeros análisis moleculares referidos a este mustélido. El objetivo principal es cuantificar el grado de variabilidad genética existente, dentro del contexto de su distribución espacial, tratando de identificar factores que podrían revelar procesos de interrupción en el flujo de genes en la población peruana de nutrias marinas.

Los resultados de este estudio fueron publicados originalmente el 19 de marzo del 2010 en la revista científica Conservation Genetics por Juan Valqui, Günther B. Hartl y Frank E. Zachos bajo el título: "Non-invasive genetic analysis reveals high levels of $m t D N A$ variability in the endangered SouthAmerican marine otter (Lontra felina)" [10] con el número de acceso por internet: DOI 10.1007/ s10592-010-0069-9. El estudio forma parte de la tesis doctoral del primer autor en el Instituto de Zoología de la Universidad Christian-Albrechts en Kiel, Alemania.

\section{METODOLOGÍA}

\section{Colecta de muestras}

El ADN de un individuo se puede obtener a partir de muestras de su tejido. La mayoría de métodos implican la captura de individuos, lo cual, aparte de representar problemas logísticos (la nutria marina es difícil de avistar y por lo tanto también de capturar), puede perturbar a los individuos de por sí huraños. Es por ello que en este estudio se decidió proceder con el análisis de ADN a partir de muestras de heces, que representa el método menos invasivo.

Entre agosto y setiembre del 2008, un equipo de cuatro personas viajó en una camioneta a lo largo de la costa peruana desde Vesique $\left(9^{\circ} 12^{\prime} \mathrm{S}\right)$ hasta el puerto de Vila Vila ( $\left.18^{\circ} 07^{\prime} S\right)$, cubriendo más de $1200 \mathrm{~km}$ de litoral dentro del rango de distribución peruano de la nutria marina. Se colectaron muestras de heces de nutrias marinas en cuatro áreas principales denominadas: Norte, Lima, Ica y Sur (fig. 2), las cuales estaban separadas por tramos de costa no evaluada, distantes a 340 $\mathrm{km}, 347 \mathrm{~km}$ y $370 \mathrm{~km}$, respectivamente. Se visitaron 25 localidades, tomándose un total de 87 muestras en 20 de ellas (fig. 2). Las heces fueron colectadas principalmente en regiones de litoral rocoso (fig. 3), dentro de cuevas (fig. 4) y sobre rocas de la orilla donde se encuentran las típicas letrinas usadas por la especie para la marcación. También se colectaron muestras de botes en puertos. Se prefirió la colecta de heces frescas, pues el ADN se degrada considerablemente después de pocos días [11]. Las muestras se colectaron usando guantes quirúrgicos (fig. 4) y, cuando fue necesario, un brazo mecánico; luego fueron puestas en etanol de $96 \%$ dentro de tubos de $50 \mathrm{ml}$ y selladas con teflón. Fueron puestas en refrigeración, para lo cual se portaba una caja isotérmica con hielo (fig. 4). Entre la colecta y la refrigeración no pasaban más de 10 minutos. Después de obtener los permisos debidos para su exportación (aproximadamente dos meses), las muestras fueron llevadas a los laboratorios del Instituto de Zoología de la Universidad Christian-Albrechts en Kiel, Alemania.

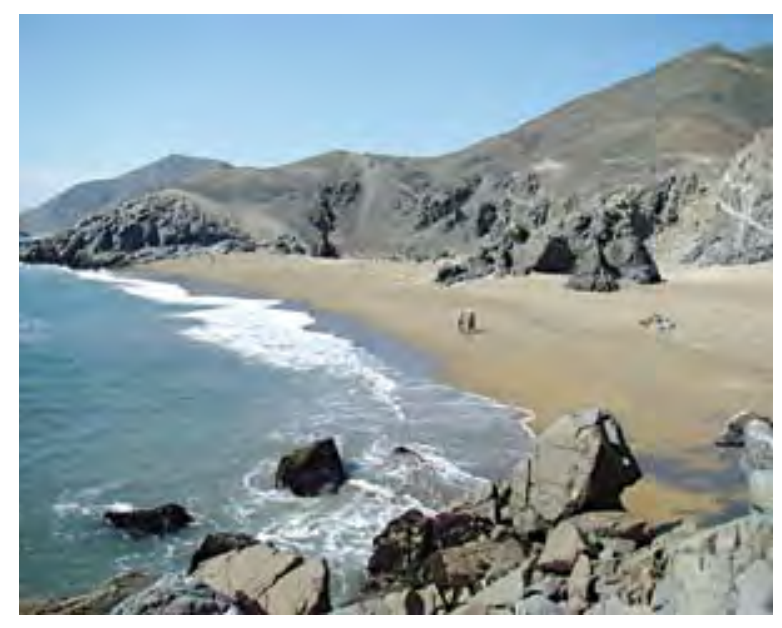

Figura 3: Litoral peruano

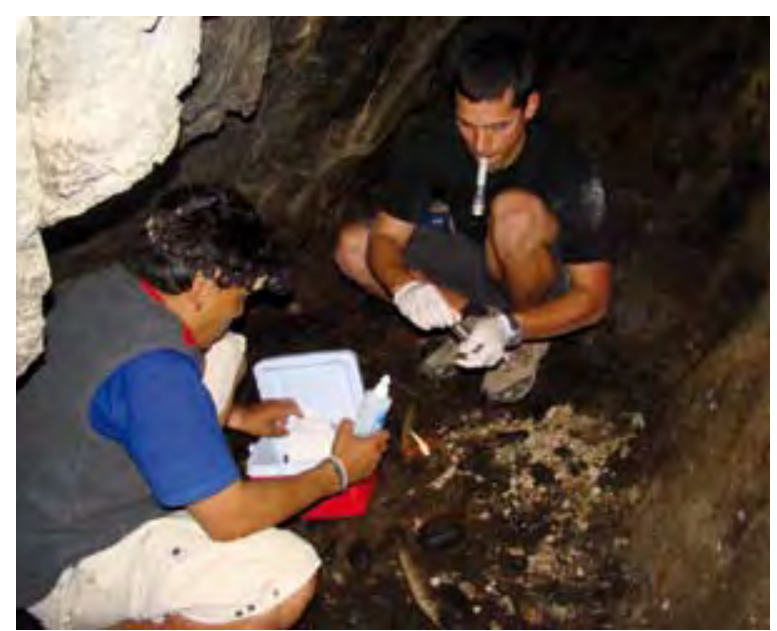

Figura 4: Colecta de muestras en cueva 


\section{Procesamiento y análisis de muestras}

En el laboratorio se procedió a la extracción del ADN mediante el QIAMP Stool Minikit y a la multiplicación de los genes estudiados mediante la reacción en cadena de la polimerasa (PCR, por sus siglas en inglés). Los productos del PCR fueron secuenciados mediante el secuenciador automático $M e g a B A C E$ y las secuencias obtenidas fueron alineadas y analizadas mediante el programa Bioedit [12] y colapsadas en haplotipos con el programa FaBox [13]. Así como es posible que en este análisis dos secuencias (haplotipos) idénticas vengan de dos individuos diferentes (por ejemplo, dos individuos cercanamente emparentados pueden compartir un haplotipo en genes mitocondriales), también se tiene que considerar la posibilidad de obtener pseudoréplicas, es decir que dos muestras diferentes con secuencias idénticas provengan de un mismo individuo. En este análisis se procedió de manera conservadora y, considerando el rango de los individuos, se descartaron los haplotipos idénticos que procedieran de menos de cinco kilómetros de distancia.

Con el programa DnaSP [14] se calcularon las siguientes medidas de diversidad:

- La diversidad de haplotipos, que es la medida de singularidad de un haplotipo particular en una población. Su valor (h) está relacionado a la frecuencia de cada haplotipo presente en la muestra y al tamaño de ésta. $\mathrm{h}$ describe la presencia de diferencias (variabilidad genética), pero no detalla en qué medida está presente.

- La diversidad de nucleótidos, que mide el grado de polimorfismo (variación) genético dentro de una población. Su valor $(\pi)$ está relacionado al número promedio de diferencias de los nucleótidos en un locus (lugar en una secuencia) particular, entre dos secuencias elegidas al azar dentro de una población. A diferencia de $h, \pi$ da una medida de variabilidad genética.

Además se utilizó el programa Arlequin [15] y se realizó un análisis molecular de variancia (AMOVA) para detectar diferenciación entre grupos muestrales. Para descubrir patrones de aislamiento por distancia se realizó un test de Mantel con dos matrices: la del número de diferencias en los nucleótidos de cada dos haplotipos y la de la distancia geográfica entre los mismos.

\section{RESULTADOS Y DISCUSIÓN}

\author{
Obtención de secuencias genéticas de 24 \\ individuos de Lontra felina
}

Cuarenta y uno de las 87 muestras tomadas lograron ser amplificadas en un fragmento de la región control del ADN mitocondrial. Cuatro de esas muestras no fueron incluidas por presentar fragmentos muy cortos, quedando 37 muestras secuenciadas. Esto significa un éxito de amplificación del 43\%, valor similar al de otros estudios basados en la extracción de $\mathrm{ADN}$ a partir de muestras de heces $[16,17]$. Posteriormente se descartaron 13 de las 37 muestras debido a que procedían de una distancia menor a cinco kilómetros de otra muestra con idéntica secuencia (posible pseudoreplicación). Por ello se contó con un número final de 24 secuencias, asumiendo que cada una proviene de un individuo diferente. La secuencia obtenida para las muestras analizadas tenía una longitud de $265 \mathrm{pb}$ (pares de bases).

\section{Primer registro de secuencias genéticas de Lontra felina en GenBank}

GenBank (http://www.ncbi.nlm.nih.gov/sites/entrez? $\mathrm{db}=$ nucleotide) es un banco de datos que contiene secuencias genéticas de más de 100000 organismos procedentes de estudios en laboratorios de todo el mundo. La comparación de las secuencias de este estudio con las secuencias existentes en GenBank, mostraron la más alta similitud con la especie Lontra canadienses, que no habita en el Perú y cuyas muestras no son trabajadas en el laboratorio usado para este estudio, por lo que se pudo descartar la posibilidad de contaminación. En el análisis de las 24 secuencias se encontraron 11 haplotipos (Lf01-Lf11) (Tabla 1), que fueron subidos al banco de datos con los números de acceso: GU982296-GU982306.

\section{Identificación de la variabilidad de haplotipos $(\mathrm{h}=\mathbf{0 . 8 6})$ y de nucleótidos $(\boldsymbol{\pi}=\mathbf{0 . 0 1 1 7})$}

La diversidad de los haplotipos (b) y de los nucleótidos $(\pi)$ calculada fue de 0,86 y 0,0117 , respectivamente. A pesar de ser una especie amenazada y presente en tres países, poco es lo que se sabe actualmente de la nutria marina y su genética poblacional. El resultado más sorprendente de este estudio es, tal vez, la obtención de una variabilidad mitocondrial relativamente alta. Con 24 individuos 
analizados, se obtuvo mayor cantidad de haplotipos en un fragmento corto de la región control del ADN mitocondrial que la obtenida en un fragmento más largo en diferentes estudios de la población de la nutria Lutra lutra, que vive en diferentes hábitats de agua dulce de Europa, Asia y África. Para esta nutria, Ferrando et al. [18] hallaron una diversidad de haplotipos y de nucleótidos de $\mathrm{h}=0,16$ y $\pi=0,0006$ respectivamente, en un fragmento de $361 \mathrm{pb}$ para la población europea. Stanton et al. [19] obtuvieron recientemente valores de $h=0,73$ y $\pi=0,003$, en $299 \mathrm{pb}$ para la población de la misma especie en el Reino Unido, mientras que Finnegan y Néill [20] obtuvieron los valores $\mathrm{h}=0,75$ en $299 \mathrm{pb}$ para su población en Irlanda. Aunque las poblaciones de Lutra lutra son sin duda mucho mayores -se registra más de 10000 individuos estimados solamente para la población del Reino Unido [2]- su diversidad de haplotipos y de nucleótidos es considerablemente más baja que la de Lontra felina.

El empobrecimiento genético en Lutra lutra es incluso mayor al de otra especie de nutria marina que habita en el Pacífico norte (Enhydra lutris), que sufrió una pérdida en el número de individuos estimada en un $99 \%$, pero siguió mostrando valores de variabilidad de haplotipos de $\mathrm{h}=0,41$ [21]. Son escasos los estudios que se ocupan de la genética de las especies del género Lontra, pero en un estudio del huillín (Lontra provocax) que habita en los ríos y lagos del sur de Chile y Argentina, sólo se obtuvo un haplotipo en el análisis de muestras de 13 individuos [17]. En la especie Lontra Longicaudis, que ocupa diferentes hábitats ribereños en Centro y Sudamérica, a partir de muestras de 20 individuos se obtuvo valores de diversidad de haplotipo $\mathrm{de} h=0.82$, que están en el rango de los valores obtenidos para Lontra felina en estudio, a la vez que se obtuvo valores de diversidad de nucleótidos mucho menores $(\pi=0,0049)$ [22]. Con este estudio se da indicios de que la nutria marina es la de mayor diversidad genética entre las especies del género Lontra en Sudamérica [10].

\section{Estructuras genéticas de la población de la nutria marina en el Perú}

En los análisis no se detectó la existencia de aislamiento por distancia ni subestructuración en el genoma mitocondrial. Esto sugeriría que una reciente interrupción en el flujo genético no ha resultado aún en una diferenciación a través de la deriva genética o confirmaría la existencia de un flujo de genes entre las localidades con presencia de la especie. Si bien los efectos de la actividad humana son postulados como causantes de la fragmentación en población de la nutria marina, la costa peruana tiene un carácter naturalmente fragmentado. Aunque se asume que el rango individual comprende menos de cinco kilómetros [5], la nutria podría presentar migraciones frecuentes en por lo menos algunos individuos. La adaptación a su medio fragmentado puede incluir una adaptación evolutiva de comportamiento que permite el flujo genético en esta condición de fragmentación y, por lo tanto, también representa una adaptación a un nuevo factor de fragmentación de origen antrópico.

Siete de los 11 haplotipos hallados en este estudio se encuentran en el grupo muestral Sur, mientras que en el grupo Norte solamente se encontró dos haplotipos. El haplotipo Lf08 está presente en los cuatro grupos muestrales, mientras que ocho de los 11 haplotipos están presentes en sólo uno de los grupos (tabla 1).

Tabla 1: Distribución y frecuencia de los 11 haplotipos hallados en este estudio, dentro de sus respectivas áreas de muestreo.

\begin{tabular}{|c|c|c|c|c|c|}
\hline \multirow[t]{2}{*}{ Haplotipo } & \multicolumn{4}{|c|}{ Grupo muestral } & \multirow[t]{2}{*}{ Suma } \\
\hline & $\begin{array}{c}\text { Norte } \\
\left(9^{\circ} 13^{\prime} S-10^{\circ} 05^{\prime} S\right) \\
\end{array}$ & $\begin{array}{c}\text { Lima } \\
\left(12^{\circ} 28^{\prime} S-13^{\circ} 01 \text { 'S }\right)\end{array}$ & $\begin{array}{c}\text { Ica } \\
\left(15^{\circ} 26^{\prime} \mathrm{S}-15^{\circ} 26^{\prime} \mathrm{S}\right)\end{array}$ & $\begin{array}{c}\text { Sur } \\
\left(17^{\circ} 00^{\prime} S-18^{\circ} 07^{\prime} S\right)\end{array}$ & \\
\hline Lf01 & - & - & - & 3 & 3 \\
\hline Lf02 & - & 1 & - & - & 1 \\
\hline Lf03 & - & 1 & - & - & 1 \\
\hline Lf04 & 1 & - & - & - & 1 \\
\hline Lf05 & - & 1 & - & - & 1 \\
\hline Lf06 & - & - & - & 1 & 1 \\
\hline Lf07 & - & 1 & 1 & 2 & 4 \\
\hline Lf08 & 3 & 1 & 1 & 3 & 8 \\
\hline Lf09 & - & - & - & 1 & 1 \\
\hline Lf10 & - & - & - & 1 & 1 \\
\hline Lf11 & - & 1 & - & 1 & 2 \\
\hline Suma & 4 & 6 & 2 & 12 & 24 \\
\hline
\end{tabular}




\section{Recomendaciones para determinar la subestructuración genética en la población de Lontra felina}

El hecho de no encontrar aislamiento por distancia debe ser tomado con precaución considerando la metodología, en la cual se excluyen secuencias idénticas dentro de un rango de cinco kilómetros. Presentando un punto débil en la metodología del análisis mitocondrial, por la exclusión se puede haber ignorado la presencia de linajes maternales, considerándose como un solo individuo y sesgando la percepción de cercanía geográfica y similitud genética. En el análisis, la ausencia de aislamiento por distancia sumada al número relativamente bajo de muestras analizadas dentro de cada uno de los cuatro grupos, obligó a descartar a estos como subgrupos poblacionales genéticamente diferenciados, debiendo ser considerados meramente grupos muestrales. Para el análisis de la estructuración genética de la población de la nutria marina se debe analizar un mayor número de muestras y realizar análisis de microsatélites a partir de muestras de heces. También se puede considerar realizar estudios de telemetría [23], aunque estos presentan una metodología más invasiva.

\section{Sugerencia de medidas de conservación}

Tal como figura en la Lista Roja de la IUCN [2], se predice que la población de la nutria marina irá decreciendo en los próximos años, perdiendo su variabilidad genética. Por ello se recomienda elaborar medidas de conservación basadas en estudios genéticos de la especie. El presente estudio significa el primer paso en ese sentido, en el Perú.

La Reserva Nacional Islas y Puntas Guaneras comprende un sistema de más de 20 islas y 10 puntas y tiene como objetivo proteger más de 100000 hectáreas de costa peruana. Aquí se incluye la protección de varias especies amenazadas de aves (el pingüino de Humboldt, el potoyunco, el zarcillo, el piquero), mamíferos marinos (el lobo fino, el lobo chusco, la nutria marina) y reptiles (tortugas marinas). Esta medida ayudará a proteger a algunas metapoblaciones de la nutria marina y garantizará hasta cierto punto el flujo de genes. Sin embargo, no considera la protección de la parte sur de la población peruana, donde Mangel y Alfaro-
Shigueto [24] (en el 2004) registraron regiones con elevado número de individuos: aproximadamente 90 nutrias en $55 \mathrm{~km}$ de litoral, que deberían ser consideradas en las medidas de conservación, por ejemplo creando un área protegida. Si se confirman las predicciones de disminución de la población y se detectan subestructuras genéticas en la población de la nutria marina, se podría incluso considerar la elección de individuos de zonas de alta diversidad para el repoblamiento de zonas de menor diversidad o en las cuales la especie ya no está presente.

En las medidas de conservación se debe incluir la educación ambiental y la sensibilización de la población. Para asegurar que las medidas tomadas abarquen la distribución total de la especie, estas deben realizarse en coordinación con Chile y Argentina.

\section{CONCLUSIONES}

Este trabajo ha cubierto el rango peruano de distribución de la nutria marina y representa el primer estudio genético de la nutria marina en el Perú. A pesar de que se sabe que la nutria marina ha sufrido la influencia de las actividades humanas en la costa peruana, los resultados muestran que su variabilidad genética es relativamente alta. Esto podría deberse a la adaptación de la especie a un hábitat naturalmente fragmentado, en cuya población hasta ahora no se ha podido detectar una subestructuración. Sin embargo, por el constante aumento de las amenazas para L. felina y la predicción de disminución de su población, se recomienda tanto realizar estudios que profundicen los conocimientos sobre las estructuras genéticas poblacionales, como la protección de la especie, en especial en el extremo sur de la costa peruana.

\section{AGRADECIMIENTOS}

El trabajo de campo se pudo realizar gracias al permiso de investigación $\mathrm{N}^{\circ}$ 98-2008-INRENAIFFS-DCB otorgado por el Instituto Nacional de Recursos Naturales (INRENA) del Ministerio de Agricultura del Perú y el permiso de exportación $\mathrm{N}^{\circ} 11378$ otorgado por la autoridad CITES local. El material de campo fue provisto por IDEAWILD, con la colaboración de Elisa Ruiz. Natalia Ortiz, Daniella Biffi y Jerico Solís colaboraron en el 
trabajo de campo. Ana María Haase y Holger Valqui facilitaron el almacenamiento de las muestras en Lima. Yennifer Hernández colaboró en los análisis de laboratorio. El Instituto de Zoología de la Universidad Christian-Albrechts de Kiel en Alemania, a cargo del Dr. Günther Hartl, brindó apoyo en todos los análisis de laboratorio. La ayuda de Frank Zachos fue fundamental en los análisis estadísticos. Alexis Valqui y Thomas Valqui colaboraron en la revisión del manuscrito. El autor agradece la financiación parcial del proyecto a The Society for Marine Mammology (SMM).

\section{REFERENCIAS}

[1] S. Larivière, Lontra felina. Mammalian Species 575 (1998) 1-5.

[2] Red List of Threatened Species. Version 2009.1. www.iucnredlist.org. Accedida el 21 de Julio 2009.

[3] M. Apaza, J. Valqui, J. Mangel, M. Roca, J. Alfaro, L. Santillán, J. P. Perret, G. Onton, C. Castañeda, G. Munemura, A. Tovar, Estado de Conservación de Lontra felina (Molina 1782) en la Costa Peruana. Reporte para la Comisión Permanente del Pacífico Sur, Lima (2004).

[4] J. C. Castilla, Nuevas observaciones sobre conducta, ecología y densidad de Lutra felina (Molina, 1782) (Carnívora: Mustelidae) en Chile. Museo Nacional de Historia Natural (Santiago, Chile), Publicación Ocasional, 38(1982)187-206.

[5] G. Medina-Vogel, F. Boher, G. Flores, A. Santibáńez, C. Soto-Azat, Spacing behavior of marine otters (Lontra felina) in relation to land refuges and fishery wastes in Central Chile, J Mammal. 88 (2007) 487-494.

[6] G. Medina-Vogel, L. O. Merino, R. Monsalve Alarcón, J. de A. Vianna, Coastal-marine discontinuities, critical patch size and isolation: implications for marine otter conservation. Animal Conservation. 11 (2008) 57-64.

[7] A. Parera, Las nutrias verdaderas de la Argentina. Boletín Técnico. Fundación Vida Silvestre Argentina. 21 (1996) 1-31.

[8] J. C. Castilla, I. Bahamondes, Observaciones conductuales y ecológicas sobre Lutra felina (Molina 1782) (Carnívora: Mustelidae) en las zonas central y centro-norte de Chile. Archivos de Biología y Medicina Experimentales. 12 (1979) 119-132.

[9] W. Sielfeld y J. C. Castilla, Estado de conservación y conocimiento de las nutrias en Chile. Estudios Oceanolóogicos. 18 (1999) 69-79.
[10] J. Valqui, G. B. Hartl, F. E. Zachos, Non-invasive genetic analysis reveals high levels of mtDNA variability in the endangered South-American marine otter (Lontra felina). Conserv Genet. (2010) DOI 10.1007/s10592-010-0069-9.

[11] M. A. Murphy, K. C. Kendall, A. Robinson, L. P. Waits, The impact of time, and field conditions on brown bear (Ursus arctos) faecal DNA amplification. Conserv Genet. 8 (2007) 1219-1224.

[12] T. A. Hall, BioEdit: a user-friendly biological sequence alignment editor and analysis program for Windows 95/98/NT. Nucl Acid S. 41 (1999) 9598.

[13] P. Villesen, FaBox: an online toolbox for fasta sequences. Mol Ecol Notes 7. (2007) 965-968.

[14] J. Rozas, R. Rozas, DnaSP version 3: an integrated program for molecular population genetics and molecular evolution analysis. Bioinformatics 15 (1999) 174-175.

[15] L. Excoffier, G. Laval, S. Schneider, ARLEQUIN Ver. 3.1: An integrated software package for population genetics data analysis. Evol Bioinform Online. 1 (2005) 47-50.

[16] D. Centrón, B. Ramírez, L. Fasola, D. W. Macdonald, C. Chehébar, A. Schiavini, M.H. Cassini, Diversity of mtDNA in Southern river otter (Lontra provocax) from Argentinean Patagonia. J Hered. 99 (2008) 198-201.

[17] R. Sharma, H. Stuckas, R. Bhaskar, S. Rajput, I. Khan, S.P. Goyal, R. Tiedemann, mtDNA indicates profound population structure in Indian tiger (Panthera tigris tigris). Conserv Genet. 10 (2009) 909-914.

[18] A. Ferrando, M. Ponsà, J. Marmi, X. DomingoRoura, Eurasian otters, Lutra lutra, have a dominant mtDNA haplotype from the Iberian Peninsula to Scandinavia. J Hered. 95 (2004) 430-435.

[19] D. W. G. Stanton, G. I. Hobbs, E. A. Chadwick, F.M. Slater, M.W. Bruford, Mitochondrial genetic diversity and structure of the European otter (Lutra lutra) in Britain. Conserv Genet. 10 (2008) 733-737.

[20] L. A. Finnegan, L. O. Néill, Mitochondrial DNA diversity of the Irish otter, Lutra lutra, population. Conserv Genet. (en prensa) DOI: 10.1007/s10592009-9955-4.

[21] S. Larson, R. Jameson, M. Etnier, M. Fleming, P. Bentzen, Loss of genetic diversity in sea otters (Enhydra lutris) associated with the fur trade of the 18th and 19th centuries. Mol Ecol. 11 (2002)18991903.

[22] C. S. Trinca, H. F. Waldemarin, E. Eizirik, Genetic diversity of the Neotropical otter (Lontra longicaudis Olfers, 1818) in southern and southeastern Brazil. Braz J Biol. 67 (Suppl.) (2007) 813-818. 
[23] C. Soto-Azat, F. Boher, M. Fabry, P. Pascual, G. Medina-Vogel, Surgical Implantation of Intraabdominal Radiotransmitters in Marine Otters (Lontra felina) in Central Chile. J Wildlife Dis. 44 (2008) 979-982.
[24] J. Mangel y J. Alfaro-Shigueto, Fishing communities and marine otter (Lontra felina) conservation in Peru. En: IXth International Otter Colloquium. Resumen. 57 (2004).

E-mail: jvalqui@zoologie.uni-kiel.de 